\title{
Volatile Components in Seaweeds
}

\author{
Maria Terezinha Santos Leite Neta and Narendra Narain* \\ Federal University of Sergipe, Brazil \\ *Corresponding author: Narendra Narain, Laboratory of Flavor and Chromatographic Analyses, Federal University of Sergipe, Brazil
}

Submission: 㠿 July 03, 2018; Published: 眥 August 20, 2018

\begin{abstract}
Marine environment comprehends a large geographic area, occupying about $71 \%$ of the surface of this planet. This environment has a great diversity of plants and animals which constitute an enormous amount in the food chain, comprising of organisms living in complex habitats where they are exposed to extreme conditions, which help them to produce a wide range of potent and active substances. These are commonly not found in other environments, that results in a source of nutrients, pharmaceuticals and characteristic flavor compounds. One of these plants which are very important to the food industry is the seaweed.
\end{abstract}

\section{Introduction}

Seaweeds are a group of photosynthetic plant-like organisms that generally thrive attached to rock or other hard substrata in coastal areas [1-5]. They have high amounts of macronutrients (proteins, carbohydrates and lipids) and thus characterize for important nutritional value $[6,7]$. Seaweeds are also sources of bioactive and antioxidant compounds, besides their extracts can have antihypertensive and anti-inflammatory properties [8-11]. Another important characteristic of seaweeds is their volatile compounds profile, which plays an important role in marine ecosystems, including in the chemical communication. Volatile compounds in seaweeds act as sexual pheromones, chemical defenses against herbivores and inhibitors of bacteria and fungi [12-14]. Some volatile compounds present in seaweeds such as ammonia, methylamine, and trimethylamine are generated by microorganisms or by chemical reactions. Processes like roasting are responsible for the formation of volatile compounds, especially dimethyl sulfide which stimulates the aroma of edible green and red seaweeds, which are very much appreciated by Japanese. Other volatile compounds could act like a protection for the seaweeds, for example, the diterpene holohedral, which defends seaweeds against herbivorous fishes and invertebrates [15].

The volatile compounds of some edible seaweeds have been analyzed by GC-MS systems and it showed that volatile compounds depend on the species, its geographical origin, processing method and environmental conditions $[16,17]$. In relation to the species, some studies revealed that green seaweed produces mainly cymopole, a product of condensation of reaction between bromophenols and monoterpenes. Brown seaweeds produce sulfur compounds, $\mathrm{C}_{11}$-hydrocarbons and diterpenes while red seaweeds are known to produce halogenated compounds containing bromine and iodine, which contribute to a sweet note in the aroma of this specie of seaweeds. Organic compounds present in seaweeds also function as therapeutic drugs, flavoring agents, odorants in perfume industry and indicator of water pollutants. For these reasons, many studies were performed with the aim of determining the volatiles from seaweeds using different analytical methods as shown in the Table 1 which cites the volatile compounds identified in brown, green and red seaweeds [18,19]. Some compounds have great impact on the aroma of seaweeds and are considered "character-impact compounds", and these include iodoethane, iodopentane and isoprene (2-methyl-1-3-butadiene) present especially in red seaweeds [20]. Volatile halogenated compounds such as dibromoethane, bromoform, dibromochloromethane, bromodichloromethane and chloroiodomethane contribute to the characteristic aroma of the seaweeds with notes like marine, crustacean and green. Moreover, these compounds play an important role in chemical defense against predators [21,22].

The profile of volatile compounds can be very diverse between the various types of seaweeds [23]. This diversity is occasioned due to different species that can be capable of producing a variety of volatile compounds. The detailed identification of such compounds is very important to elucidate their aroma properties of the final product $[24,25]$. In the green seaweeds, a higher number of compounds have been identified in comparison to brown and red seaweeds (Table 1). The diversity of volatiles compounds presents in seaweeds produce different odors which involve main notes defined like "marine" and "seafood". Showed that other odor notes like "fatty", "fish", "licorice", "spices", "green", "honey" were detected in species of green, red and brown seaweeds. In these species, carboxylic acids were related to odor notes such as of licorice, honey 
and spices, while esters and Sulphur compounds were related to green odor. Notes like fish odor, marine odor, fatty odor, seafood odor are related to presence of amines and pyridines. Important volatiles responsible for the odor of green marine algae [26]. In a recent study it is reported that compounds when present in higher concentrations contribute to fruity notes in aroma characteristics of some seaweeds [27]. Another class of compounds commonly found in the aroma composition of seaweeds are the ketones. Ketones, such as $\beta$-ionone and 6-methyl-5-hepten-2-one are formed by the oxidative cleavage of carotenoids such as lycopene and phytoene [28]. These compounds are formed from the pigments contained in the seaweeds and are metabolized while capturing light and scavenging radicals [29]. $\beta$-ionone is a potent odorant in seafood and has been found in all three types of seaweeds. Other ketone frequently found in aroma of seaweeds which have an odor impact is 6-methyl-5-hepten-2-one. This ketone is also found in other foods, such as tomato as a metabolite of lycopene which results in compounds having more pleasant odors [30-32].

\section{Table 1}

\begin{tabular}{|c|c|c|c|c|}
\hline \multirow[t]{2}{*}{ Volatile compounds } & \multirow[t]{2}{*}{ Odor description* } & \multicolumn{3}{|c|}{ Types of microalgae } \\
\hline & & Green & Brown & Red \\
\hline \multicolumn{5}{|c|}{ Aldehydes } \\
\hline (E)-2-Heptenala & Green, pungent & $\mathrm{x}$ & $\mathrm{x}$ & $\mathrm{X}$ \\
\hline (E)-2-Hexenalf,i & Green, banana & $\mathrm{X}$ & $\mathrm{x}$ & $\mathrm{X}$ \\
\hline (E)-2-Octenale, $\mathrm{f}$ & Fresh, cucumber, green & $\mathrm{X}$ & $\mathrm{x}$ & $\mathrm{X}$ \\
\hline (E)-2-nonenale & Fatty, green & $\mathrm{X}$ & & \\
\hline (E)-2-Butenala & flowery & $\mathrm{X}$ & $\mathrm{x}$ & $\mathrm{X}$ \\
\hline (E)-2-Pentenala & Green, fruity & $\mathrm{X}$ & $\mathrm{X}$ & $\mathrm{X}$ \\
\hline (E,E)-2,4-decadienala,e & Fatty, cucumber & $\mathrm{X}$ & & \\
\hline (E,E)-2,4-Heptadienala & Green, fatty & $\mathrm{X}$ & $\mathrm{X}$ & $\mathrm{X}$ \\
\hline (E,E)-2,6-Nonadienala, e & Green, fatty & $\mathrm{X}$ & $\mathrm{x}$ & $\mathrm{X}$ \\
\hline (Z)-4-Heptenala & Green, fatty & $\mathrm{X}$ & $\mathrm{x}$ & $\mathrm{X}$ \\
\hline (Z)-8-Heptadecenal & - & $\mathrm{x}$ & & \\
\hline (Z,E)-2,4-Heptadienala, j & Fried & $\mathrm{X}$ & $\mathrm{x}$ & $\mathrm{X}$ \\
\hline (Z,Z)-8,11-Heptadecadienale & - & $\mathrm{x}$ & & \\
\hline (Z,Z)-9,12-Octadecadienale & - & $\mathrm{X}$ & & \\
\hline (Z,Z,Z)-8,11,14-Heptadecatrienale & Seaweed & $\mathrm{X}$ & & \\
\hline (Z,E)-2.6-Nonadienala & Green & $\mathrm{X}$ & & \\
\hline 2,4-Decadienale & Fatty, orange & $\mathrm{x}$ & & \\
\hline 2-Methyl-2-propenala & Fresh & $\mathrm{X}$ & $\mathrm{x}$ & $\mathrm{X}$ \\
\hline 2-Heptenald & Green, fatty & $\mathrm{X}$ & & \\
\hline 3-Methylbut-2-enalb & Fruity & $\mathrm{X}$ & $\mathrm{x}$ & \\
\hline 3-Methylbutanala,b, i & Peach-like & $\mathrm{X}$ & $\mathrm{x}$ & $\mathrm{X}$ \\
\hline 2-Methylbutanala & Chocolate & $\mathrm{X}$ & $\mathrm{x}$ & $\mathrm{X}$ \\
\hline 2,4-Heptadienald & Green, fruity & $\mathrm{x}$ & & \\
\hline 4-Heptenalc & Green & & & $\mathrm{X}$ \\
\hline Benzaldehydea,b, d, f & Fruity & $\mathrm{X}$ & $\mathrm{x}$ & $\mathrm{X}$ \\
\hline Ethanala & Ethereal & $\mathrm{X}$ & $\mathrm{x}$ & $\mathrm{X}$ \\
\hline Butanala & Chocolate, cocoa & $\mathrm{X}$ & $\mathrm{x}$ & $\mathrm{X}$ \\
\hline Decanala,b & Sweet, aldehydic & & & $\mathrm{X}$ \\
\hline Heptanala, f, j & Green, fresh & $\mathrm{X}$ & $\mathrm{x}$ & $\mathrm{X}$ \\
\hline Hexanala,b, d, e, f, j & Fresh, green & $\mathrm{X}$ & $\mathrm{x}$ & $\mathrm{X}$ \\
\hline Pentadecanal & Fresh, waxy & $\mathrm{X}$ & & \\
\hline Nonanala,b, f, i & Aldehydic & $\mathrm{X}$ & $\mathrm{X}$ & $\mathrm{X}$ \\
\hline Furfurald & Sweet, woody & $\mathrm{X}$ & & \\
\hline
\end{tabular}




\begin{tabular}{|c|c|c|c|c|}
\hline Octanala, i & Aldehydic & $\mathrm{X}$ & $\mathrm{X}$ & $\mathrm{X}$ \\
\hline Octadecanalb,c & Oily & & & $\mathrm{X}$ \\
\hline$\beta$-Cyclocitrala,b, d & Tropical & $\mathrm{X}$ & $\mathrm{X}$ & $\mathrm{X}$ \\
\hline 4-Propylbenzaldehydea & - & $\mathrm{X}$ & $\mathrm{X}$ & $\mathrm{X}$ \\
\hline Pentanala & Fermented, bready & $\mathrm{X}$ & $\mathrm{X}$ & $\mathrm{X}$ \\
\hline Tridecanale & Aldehydic & $\mathrm{X}$ & & \\
\hline Perillaldehydee & Herbal & $\mathrm{X}$ & & \\
\hline 4-Ethylbenzaldehydea & Fruity, almond & $\mathrm{X}$ & $\mathrm{X}$ & $\mathrm{X}$ \\
\hline \multicolumn{5}{|l|}{ Hydrocarbons } \\
\hline 1-Hexadecenea & - & $\mathrm{X}$ & & \\
\hline 1-Nonadecenef & - & & & $\mathrm{X}$ \\
\hline 1-Pentadecenea & - & $\mathrm{X}$ & $\mathrm{X}$ & \\
\hline 1-Tetradecenea & - & $\mathrm{X}$ & & \\
\hline 3-Heptadecenec & - & & & $\mathrm{X}$ \\
\hline 4,6-Dimethyldodecanea & - & $\mathrm{X}$ & $\mathrm{X}$ & $\mathrm{X}$ \\
\hline 4,8-Dimethylundecanea & - & $\mathrm{X}$ & $\mathrm{X}$ & $\mathrm{X}$ \\
\hline Heptadecadiene & - & $\mathrm{X}$ & & \\
\hline Heptadecanea, c, d & - & $\mathrm{X}$ & $\mathrm{X}$ & $\mathrm{X}$ \\
\hline Heptadecened, f & - & $\mathrm{X}$ & & $\mathrm{X}$ \\
\hline 4-Methyldecanea & - & $\mathrm{x}$ & $\mathrm{X}$ & $\mathrm{X}$ \\
\hline 7-Heptadecenee & Alkane & $\mathrm{X}$ & & \\
\hline 4-Ethyldecanea & - & $\mathrm{X}$ & $\mathrm{X}$ & $\mathrm{X}$ \\
\hline 2,4-Dimethylhexanea & - & $\mathrm{X}$ & $\mathrm{X}$ & $\mathrm{X}$ \\
\hline 2,4-Dimethylundecane & - & $\mathrm{X}$ & $\mathrm{X}$ & $\mathrm{X}$ \\
\hline 2,4-Dimethylheptanea & - & $\mathrm{X}$ & $\mathrm{X}$ & $\mathrm{X}$ \\
\hline 2-Methylheptanea & - & $\mathrm{X}$ & $\mathrm{X}$ & $\mathrm{X}$ \\
\hline 2-Methylnonanea & - & $\mathrm{X}$ & $\mathrm{X}$ & $\mathrm{X}$ \\
\hline 2,7-Dimethyloctanea & - & & $\mathrm{X}$ & $\mathrm{X}$ \\
\hline 5-Methyldecanea & - & $\mathrm{X}$ & $\mathrm{X}$ & $\mathrm{X}$ \\
\hline Nonadecanea & - & $\mathrm{X}$ & & \\
\hline Hexadecanee & - & $\mathrm{X}$ & & \\
\hline Octanea & Gasoline & $\mathrm{X}$ & $\mathrm{X}$ & $\mathrm{X}$ \\
\hline Octadecenee & - & $\mathrm{X}$ & & \\
\hline Nonanea & Gasoline & & $\mathrm{X}$ & $\mathrm{X}$ \\
\hline Hexadecenee & - & $\mathrm{X}$ & & \\
\hline Tetradecanee & Mild, waxy & $\mathrm{X}$ & & \\
\hline Tetradecenee & - & $\mathrm{X}$ & & \\
\hline Tridecanee & - & $\mathrm{X}$ & & \\
\hline Pentadecanea & Waxy & $\mathrm{X}$ & $\mathrm{X}$ & \\
\hline Pentadecenee & - & $\mathrm{X}$ & & \\
\hline \multicolumn{5}{|l|}{ Ketones } \\
\hline 2-Octanonea & Earthy & $\mathrm{X}$ & & \\
\hline 3-Octen-2-onea & Earthy, spicy & $\mathrm{X}$ & $\mathrm{X}$ & $\mathrm{X}$ \\
\hline$\alpha$-Iononea, $\mathrm{f}$ & Floral & $\mathrm{X}$ & & $\mathrm{X}$ \\
\hline$\gamma$-Butyrolactonea & Cream, caramellic & $\mathrm{X}$ & $\mathrm{X}$ & $\mathrm{X}$ \\
\hline
\end{tabular}




\begin{tabular}{|c|c|c|c|c|}
\hline$\gamma$-Hexalactonea & Herbal, coconut & $\mathrm{X}$ & $\mathrm{X}$ & $\mathrm{X}$ \\
\hline$\gamma$-Valerolactonea & Herbal, sweet & & $\mathrm{X}$ & $\mathrm{X}$ \\
\hline ß-iononea,b,d,e & Floral & $\mathrm{X}$ & $\mathrm{X}$ & $\mathrm{X}$ \\
\hline (E,E)-3,5-Octadien-2-onea & Fruity, green & $\mathrm{X}$ & $\mathrm{X}$ & $\mathrm{X}$ \\
\hline (E,Z)-3,5-Octadien-2-onea & Herbal & $\mathrm{X}$ & $\mathrm{X}$ & $\mathrm{X}$ \\
\hline 1-Octen-3-onea & Herbal, mushroom & $\mathrm{X}$ & $\mathrm{X}$ & $\mathrm{X}$ \\
\hline 1-Phenylethanonea & Floral, sweet & $\mathrm{X}$ & $\mathrm{X}$ & $\mathrm{X}$ \\
\hline 2,3-Butanedionea & Buttery, sweet & $\mathrm{X}$ & $\mathrm{X}$ & $\mathrm{X}$ \\
\hline 2,3-Octanedionef & Dill, aspargus & & & $\mathrm{X}$ \\
\hline 2,5-Octadionea & - & $\mathrm{X}$ & $\mathrm{X}$ & $\mathrm{X}$ \\
\hline 2-Butanonea & Etheral, acetone & $\mathrm{X}$ & $\mathrm{X}$ & $\mathrm{X}$ \\
\hline 2,2,6-Trimethylcyclohexanonea & Thujonic & $\mathrm{X}$ & $\mathrm{X}$ & $\mathrm{X}$ \\
\hline 2-Heptanonea, $\mathrm{f}$ & Fruity, spicy & $\mathrm{X}$ & $\mathrm{X}$ & $\mathrm{X}$ \\
\hline 2,6,6-Trimethyl-2-hydroxycyclohexanonea & Sweet, herbal & $\mathrm{X}$ & & \\
\hline 2-Pentadecanonec & Floral, fresh, jasmin & & & $\mathrm{X}$ \\
\hline 2-Pentanonea & Sweet, fruity & $\mathrm{X}$ & $\mathrm{X}$ & $\mathrm{X}$ \\
\hline 3-Hydroxy-2-butanonea & Sweet, buttery & $\mathrm{X}$ & $\mathrm{x}$ & $\mathrm{X}$ \\
\hline 3-Penten-2-onea & Fruity, acetone & $\mathrm{X}$ & $\mathrm{X}$ & $\mathrm{X}$ \\
\hline 6-Methyl-3,5-heptadien-2-onea & Spicy, cinnamom & & $\mathrm{x}$ & $\mathrm{X}$ \\
\hline 6-Methyl-5-hepten-2-onea, d & Citrus, green & $\mathrm{X}$ & $\mathrm{X}$ & $\mathrm{X}$ \\
\hline 5-Ethyl-2(5H)-furanonea & Caramel & $\mathrm{X}$ & $\mathrm{x}$ & $\mathrm{X}$ \\
\hline trans- $\beta$-Iononea & Floral, sweet & $\mathrm{X}$ & $\mathrm{x}$ & $\mathrm{X}$ \\
\hline \multicolumn{5}{|l|}{ Alcohols } \\
\hline (E)-2-Ethyl-1-hexanola & Citrus, fresh & $\mathrm{X}$ & $\mathrm{X}$ & $\mathrm{X}$ \\
\hline (E)-2-Penten-1-ola, e & Mushroom & $\mathrm{X}$ & $\mathrm{X}$ & $\mathrm{X}$ \\
\hline (Z)-1,5-Octadien-3-ol & Green, marine & $\mathrm{X}$ & $\mathrm{X}$ & $\mathrm{X}$ \\
\hline (Z)-2-Ethyl-1-hexanola & Citrus & & $\mathrm{X}$ & \\
\hline (Z)-2-Penten-1-ola & Green & $\mathrm{X}$ & $\mathrm{X}$ & $\mathrm{X}$ \\
\hline 1,7-Octadien-3-ola & - & & $\mathrm{X}$ & \\
\hline 1-Hexadecanolc & Waxy, green & & & $\mathrm{X}$ \\
\hline 1-Hexanola,b & Herbal & $\mathrm{X}$ & $\mathrm{X}$ & $\mathrm{X}$ \\
\hline 1-Octanola,f & Waxy, green & $\mathrm{X}$ & $\mathrm{X}$ & $\mathrm{X}$ \\
\hline 1-Octen-3-ol & Earthy, mushroom & $\mathrm{X}$ & $\mathrm{X}$ & $\mathrm{X}$ \\
\hline 1-Pentanola & Fermented, oily, sweet & $\mathrm{X}$ & $\mathrm{X}$ & $\mathrm{X}$ \\
\hline 1-Penten-3-ola, i & Green & $\mathrm{X}$ & $\mathrm{X}$ & $\mathrm{X}$ \\
\hline 2-(2-Methoxypropoxy)-1-propanola & - & $\mathrm{X}$ & $\mathrm{X}$ & $\mathrm{X}$ \\
\hline 2,3-Butanediola & Fruity, creamy & & & $\mathrm{X}$ \\
\hline 2-Methyl-3-pentanola & - & $\mathrm{X}$ & $\mathrm{X}$ & $\mathrm{X}$ \\
\hline 2-Octen-1-ola & Green, vegetable & $\mathrm{X}$ & $\mathrm{X}$ & $\mathrm{X}$ \\
\hline 2-Penten-l-ola & Green & $\mathrm{X}$ & & \\
\hline 3-Pentanole & Herbal & $\mathrm{X}$ & $\mathrm{X}$ & $\mathrm{X}$ \\
\hline Isophytolc & Floral, herbal & & & $\mathrm{X}$ \\
\hline Benzyl alcohold & Floral, rose & $\mathrm{X}$ & & $\mathrm{X}$ \\
\hline Perillyl alcohole & - & $\mathrm{X}$ & & \\
\hline Phytolc & Floral, balsamic & $\mathrm{X}$ & & $\mathrm{X}$ \\
\hline
\end{tabular}




\begin{tabular}{|c|c|c|c|c|}
\hline Cedrolc & Woody & & & $\mathrm{X}$ \\
\hline ß-terpineole & Woody & $\mathrm{X}$ & & \\
\hline \multicolumn{5}{|l|}{ Esters } \\
\hline 2-Propenoic acida & - & $\mathrm{X}$ & & \\
\hline 3-Methylbutanoic acida & Cheesy & $\mathrm{X}$ & $\mathrm{X}$ & $\mathrm{X}$ \\
\hline Butanoic acida & Cheesy & $\mathrm{X}$ & $\mathrm{X}$ & $\mathrm{X}$ \\
\hline Ethanoic acida & Acidic & $\mathrm{X}$ & $\mathrm{X}$ & $\mathrm{X}$ \\
\hline Ethyl acetatec & Fruity, sweet & $\mathrm{X}$ & & $\mathrm{X}$ \\
\hline Hexanoic acida,e & Fatty, cheesy & $\mathrm{X}$ & $\mathrm{X}$ & $\mathrm{X}$ \\
\hline Heptanoic acida & Cheesy, rancid & $\mathrm{X}$ & $\mathrm{X}$ & $\mathrm{X}$ \\
\hline Methyl hexanoatec & Waxy fatty & $\mathrm{X}$ & & $\mathrm{X}$ \\
\hline 2-Methylpropanoic acida & Acidic & $\mathrm{X}$ & $\mathrm{X}$ & $\mathrm{X}$ \\
\hline 2-propenoic acid,2-methyl dodecylesterc & - & & & $\mathrm{X}$ \\
\hline Methyl linolenate & - & $\mathrm{X}$ & & \\
\hline Methyl tetradecanoatec & Waxy, fatty & $\mathrm{X}$ & & \\
\hline Nonanoic acidd & Waxy, dirty & $\mathrm{X}$ & & \\
\hline Ethyl hexanoatea & Fruity, pineapple & & & $\mathrm{X}$ \\
\hline Octanoic acida & Fatty, waxy & $\mathrm{X}$ & $\mathrm{X}$ & $\mathrm{X}$ \\
\hline Octyl acetatee & Floral, green & $\mathrm{X}$ & & \\
\hline Isobutyl acetate & Fruity, sweet & $\mathrm{X}$ & & \\
\hline \multicolumn{5}{|l|}{ Sulfur compounds } \\
\hline Dimethyl sulfidea,d,e,c & Sulfurous, onion, sweet & $\mathrm{X}$ & $\mathrm{X}$ & $\mathrm{X}$ \\
\hline Dimethyl sulfoxidea,d & Fatty, oily & $\mathrm{X}$ & $\mathrm{X}$ & $\mathrm{X}$ \\
\hline Dimethyl trisulfidec & Sulfurous, onion & & & $\mathrm{X}$ \\
\hline \multicolumn{5}{|l|}{ Other compounds } \\
\hline 2-Iodo-propanea & - & & $\mathrm{X}$ & \\
\hline Iodo-ethanea & - & & $\mathrm{X}$ & \\
\hline Iodo-methanea & - & & $\mathrm{X}$ & \\
\hline 1-Iodo-pentanea,b & - & $\mathrm{X}$ & $\mathrm{X}$ & $\mathrm{X}$ \\
\hline 1-Iodo-octanea & - & & $\mathrm{x}$ & \\
\hline 2-Ethylfurana & Sweet, burnt & $\mathrm{X}$ & $\mathrm{X}$ & $\mathrm{X}$ \\
\hline Germacrene Db & - & & $\mathrm{X}$ & \\
\hline 2-Pentylfurana,f & Fruity, green & $\mathrm{X}$ & $\mathrm{X}$ & $\mathrm{X}$ \\
\hline m-Cymenee & - & $\mathrm{X}$ & & \\
\hline 2-Methylfurana & Chocolate & $\mathrm{X}$ & $\mathrm{X}$ & $\mathrm{X}$ \\
\hline Trans-2-(2-pentenyl)furanf & - & & & $\mathrm{X}$ \\
\hline Tribromomethaneh,i & - & $\mathrm{X}$ & $\mathrm{X}$ & \\
\hline
\end{tabular}

A large quantity of compounds present in the aroma of seaweeds is due to the significant contribution of lipid-derived volatiles, such as aldehydes and alcohols, which relate to the non-appreciable aroma characteristics of microalgae. It is for this reason that prevention and control of lipid oxidation is recommended to ensure good flavor quality of the product [33]. In some species of seaweed it is possible to find compounds which belong to furans. This class consists of a family of important chemicals in the formation of crab flavor and are sourced from the oxidative degradation of linolenic acid [34]. The aroma diversity of seaweeds is a result of the variety and concentration range of the aroma compounds. Since microalgae could possess important odor characteristics, it can be used in food and feed formulations. Some of these aroma characteristics may be desirable in some applications but unwanted in others. For example, while sulfur-containing compounds are highly appreciated in certain foods such as oysters, roasted coffee, fried meat, toasted bread or cheddar cheese, these can lead to very irritating offflavors, for example, in milk $[35,36]$. This review reveals that the odor characteristics of seaweeds can be very distinct, and it varies between the three types: red, brown and green. Ulva sp., a specie 
of green seaweed, received notes like seaweed odor, marine odor and seafood odor. However, Laminaria digitate, brown seaweed, showed stronger seaweed aroma and milder honey aroma [37] and the red seaweeds received the highest scores for the seaweed odor attribute.

\section{Conclusion}

The volatile profiles of brown, green and red microalgae have been presented in this review on seaweeds. Volatile compounds in seaweeds also act as sexual pheromones and inhibitors of bacteria and fungi. Moreover, the specific aroma notes of these seaweeds as reported in publications are also detailed. Among the three types of seaweeds, the green has been studied much more and relatively a large number of volatile compounds have been identified in this seaweed as compared to brown and red types. The characteristic marine odor is much more appreciated in food preparations and is very prominent in green seaweed.

\section{References}

1. Costa Lotufo LV, Wilke DV, Jimenez PC, Epifanio RA (2009) Organismos marinhos como fontes de novos fármacos e perspectivas. Química Nov 32: 703-716.

2. Gressler V, Colepicolo P, Pinto E (2009) Useful strategies for algal volatile analysis. Current Analytical Chemistry 5(3): 271-292.

3. Blunt JW, Copp BR, Keyzers RA, Munro MHG, Prinsep MR (2015) Marine natural products. Nat Prod Rep 32(2): 116-211.

4. Hamed I, Özogul F, Özogul Y, Regenstein JM (2015) Marine bioactive compounds and their health benefits: a review. Comprehensive Reviews in Food Science and Food Safety 14(4): 446-465.

5. Narain N, Nunes ML (2007) Marine animal and plant products, in handbook of meat, poultry \& seafood quality. Blackwell Publishing. $\left(1^{\text {st }}\right.$ edn). USA.

6. Szabo NJ, Matulka, RA, Kiss L, Licari P (2012) Safety evaluation of a high lipid whole algalin flour (WAF) from Chlorella protothecoides. Regulatory Toxicology and Pharmacology 63(1): 155-165.

7. Robertson RC, Mateo, MRG, Grady MN, Guiheneuf F, et al. (2016) An assessment of the techno-functional and sensory properties of yoghurt fortified with a lipid extract from the microalga Pavlova lutheri. Innovative Food Science and Emerging Technologies 37(2): 237-246.

8. Balboa EM, Conde, Moure A, Falqué E, Domínguez H (2013) In vitro antioxidant properties of crude extracts and compounds from brown algae. Food Chemistry 138(2-3): 1764-1785

9. Gómez Ordóñez E, Jiménez Escrig A, Rupérez P (2010) Dietary fibre and physicochemical properties of several edible seaweeds from the northwestern Spanish coast. Food Research International 43(9): 22892294.

10. Gupta S, Abu Ghannam N (2011) Recent developments in the application of seaweeds or seaweed extracts as a means for enhancing the safety and quality attributes of foods. Innovative Food Science \& Emerging Technologies 12: 600-609.

11. Samarakoon K, Jeon YJ (2012) Bio-functionalities of proteins derived from marine algae-a review. Food Research International 48(2): 948960.

12. Akakabe Y, Iwamoto S, Miyamura S, Kajiwara T (2007) Induction of (Z)8- heptadecene in marine green alga Bryopsis maxima by mechanical wounding. Fisheries Science 73(5): 1057-1060.

13. Amsler CD, Fairhead VA (2005) Defensive and sensory chemical ecology of brown algae. Advances in Botanical Research 43: 1-91.
14. López Pérez O, Picon A, Nuñez M (2016) Volatile compounds and odour characteristics of seven species of dehydrated edible seaweeds. Food Research International 99(3): 1002-1010.

15. Ito K, Hori K (1989) Seaweed: chemical composition and potential food uses. Food Rev International 5(1): 101-144.

16. Casais FP, Yusty MAL, Quirós AR, Hernández LJ (2013) Rapid identification of volatile compounds in fresh seaweed. Talanta 115: 798800 .

17. Pape MAL, Priol JG, Prost C, Demaimay M (2004) Optimization of dynamic headspace extraction of the edible red algae Palmaria palmata and identification of the volatile components. Journal of Agricultural and Food Chemistry 52(3): 550-556.

18. Kenessov B, Koziel JA, Bakaikina NV, Orazbayeva D (2016) Perspectives and challenges of on-site quantification of organic pollutants in soils using solid-phase microextraction. Trends Anal Chem 85(1): 111-122.

19. Sun SM, Chung GH, Shin TS (2012) Volatile compounds of the green alga, Capsosiphon fulvescens. Journal Applied Phycol 24(5): 1003-1013.

20. Broadgate WJ, Malin G, Küpper FC, Thompson, A, Liss PS (2004) Isoprene and other non-methane hydrocarbons from seaweeds: A source of reactive hydrocarbons to the atmosphere. Marine Chemistry 88(1-2): $61-73$

21. Pape MA l, Priol GJ, Demaimay M (2006) Effect of two storage conditions on the odor of an edible seaweed, Palmaria palmata, and optimization of an extraction procedure preserving its odor characteristics. J Food Science 67 (8): 3135-3139.

22. Lanturnus F, Wienck C, Kliiser H (1996) Antartic macroalgae-sources of volatiles halogenated organic compounds. Marine Environment 41(2): 169-181.

23. Maruti A, Guerrero DE, Barroso CC, Castro R (2018) Optimization of a multiple headspace sorptive extraction method coupled to gas chromatography-mass spectrometry for the determination of volatile compounds in macroalgae. Journal of Chromatography A 1551: 41-51.

24. Santos AB, Fernandes AS, Wagner R, Lopes JE, Zepka QL (2016) Biogeneration of volatile organic compounds produced by Phormidium autumnale in heterotrophic bioreactor. Journal of Applied Phycology 28(3): 1561-1570.

25. Zhou LV, Chen J, Xu J, Li Y, Zhoua C, et al. (2016). Change of volatile components in six microalgae with different growth phases. Journal of the Science of Food and Agriculture 97(3): 761-769.

26. Sugisawa H, Nakamura K, Tamura H (1990) The aroma profile of the volatiles in marine green algae (Ulva Pertusa). Food Rev Int 6(4): 573589.

27. Durme VJ, Goiris K, Winne DA, Cooman DL, Muylaert K (2013) Evaluation of the volatile composition and sensory properties of five species of microalgae. J Agric Food Chem 61(46): 10881-10890.

28. Firouzi J, Gohari AR, Rustaiyan, A, Larijani K, Saeidnia S (2013) Composition of the essential oil of Nizamuddinia zanardinii, a Brown alga collected from Oman Gulf. J Essential Oil Bear Plants 16(5): 689692.

29. Yamamoto M, Baldermann S, Yoshikawa K, Fujita N, Mase $\mathrm{N}$, et al (2014) Determination of volatile compounds in four commercial samples of Japanese green algae using solid phase microextraction gas chromatography mass spectrometry, Science World J 2014: 1-8.

30. Michalak I, Tuhy L, Chojnacka K (2015) Seaweed extract by microwave assisted extraction as plant growth biostimulant. Open Chem 13(1) 1183-1195.

31. Höckelmann C, Jüttner F (2005) Off-flavours in water: hydroxyketones and betaionone derivatives as new odour compounds of freshwater cyanobacteria. Flavour and Fragrance Journal 20(4): 387-394. 
32. Milovanovic I, Misan A, Simeunovit J, Kovac D, Jambrec D (2015) Determination of volatile organic compounds in selected strains of cyanobacteria. Journal of Chemistry 969542 pp.6.

33. Hosoglu MI (2018) Aroma characterization of five microalgae species using solid-phase microextraction and gas chromatography-mass spectrometry/olfactometry. Food Chemistry 240(1): 1210-1218.

34. Gu SQ, Wang XC, Tao NP, Wu N (2013) Characterization of volatile compounds in different edible parts of steamed Chinese mitten crab (Eriocheir sinensis). Food Research International 54(1): 81-92.

35. Glover KE, Budge S, Rose M, Rupasinghe H, Maclaren L, et al. (2012) Effect of feeding fresh forage and marine algae on the fatty acid composition and oxidation of milk and butter. Journal of Dairy Science 95(6): 2797 2809

36. Zhang Z, Li TL, Wang D, Zhang, L, Chen G (2009) Study on the volatile profile characteristics of oyster Crassostrea gigas during storage by a combination sampling method coupled with GC/MS. Food Chemistry, 115(3): 1150-1157.

37. Michel F, Priol J, Galaup P, Demaimay M (1997) Effect of two drying techniques on the volatile compounds of two alimentary algae, Ulva sp. and Palmaria palmate, Sciences des Aliments 17: 601-617.
Creative Commons Attribution 4.0 International License

For possible submissions Click Here

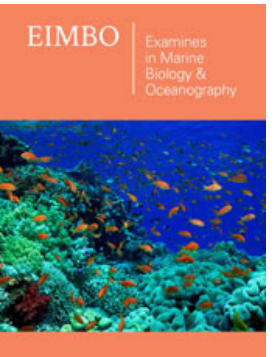

\section{Examines in Marine Biology \& Oceanography}

\section{Benefits of Publishing with us}

- High-level peer review and editorial services

- Freely accessible online immediately upon publication

- Authors retain the copyright to their work

- Licensing it under a Creative Commons license

- Visibility through different online platforms 